\title{
Principles of Local Drug Delivery to the Inner Ear
}

\author{
Alec N. Salt ${ }^{a}$ Stefan K. Plontke ${ }^{b}$ \\ a Department of Otolaryngology, Washington University School of Medicine, St. Louis, Mo., USA; \\ ${ }^{b}$ Department of Otorhinolaryngology, Head and Neck Surgery and Tübingen Hearing Research Center, \\ University of Tübingen, Tübingen, Germany
}

\section{Key Words}

Animal $\cdot$ Cochlea $\cdot$ Perilymph · Controlled release .

Inner ear · Human • Local drug delivery • Pharmacokinetics

\begin{abstract}
As more and more substances have been shown in preclinical studies to be capable of preventing damage to the inner ear from exposure to noise, ototoxic drugs, ischemia, infection, inflammation, mechanical trauma and other insults, it is becoming very important to develop feasible and safe methods for the targeted delivery of drugs to specific regions in the inner ear. Recently developed methods for sampling perilymph from the cochlea have overcome major technical problems that have distorted previous pharmacokinetic studies of the ear. These measurements show that drug distribution in perilymph is dominated by passive diffusion, resulting in large gradients along the cochlea when drugs are applied intratympanically. Therefore, in order to direct drugs to specific regions of the ear, a variety of delivery strategies are required. To target drugs to the basal cochlear turn and vestibular system while minimizing exposure of the apical cochlear turns, single one-shot intratympanic applications are effective. To increase the amount of drug reaching the apical cochlear turns, repeated intratympanic injections or controlled-release drug delivery systems, such as biodegradable biopolymers or catheters and pumps,
\end{abstract}

are more effective. However, if the applied substance does not easily pass through the round window membrane, or if a more widespread distribution of drug in the ear is required, then intralabyrinthine injections of the substance may be required. Intralabyrinthine injection procedures, which are currently in development in animals, have not yet been proven safe enough for human use.

Copyright $\odot 2009$ S. Karger AG, Basel

\section{Introduction}

Local drug delivery to the inner ears of humans was first used more than half a century ago for the treatment of Ménière's disease with local anesthetics [1,2] and antibiotics [3]. It was popularized in the 1990s as it became accepted that locally applied gentamicin provided an effective treatment for the vestibular symptoms of patients with Ménière's disease with limited risk to hearing [4-6]. In addition to aminoglycosides and anesthetics, a variety of drugs have been applied extracochlearly to the round window niche in humans, including neurotransmitters

A.N.S. and S.K.P. contributed equally to this paper. A.N.S. is a member of the scientific advisory board of Otonomy; however, this work was not supported by Otonomy.

\section{KARGER}

(๑) 2009 S. Karger AG, Basel

Fax +4161306 1234

E-Mail karger@karger.ch

www.karger.com
Accessible online at: www.karger.com/aud
Dr. Alec N. Salt

Department of Otolaryngology, Box 8115

Washington University School of Medicine

660 South Euclid Avenue, St. Louis, MO 63110 (USA)

Tel. +1 314362 7560, Fax +1 314362 1618, E-Mail salta@ent.wustl.edu 
and neurotransmitter antagonists for tinnitus [7], monoclonal antibodies for autoimmune inner ear disease [8] or apoptosis inhibitors (AM-111) for noise-induced hearing loss [9]. However, glucocorticoids have become the most widely used drugs for local application to the inner ear, and have been given to treat Ménière's disease [10], idiopathic sudden sensorineural hearing loss [11-13], autoimmune inner ear disease [14] and tinnitus [15], even though the evidence supporting their use is rather limited [16, 17]. Nevertheless, at present, dosing protocols and the selection of drug delivery systems are almost totally empirically based, and there is still only a limited understanding of the pharmacokinetics of drugs in the ear.

\section{Pharmacokinetics of the Inner Ear}

Although the 'LADME' scheme was developed to describe the pharmacokinetic processes in the human body following a given dosage regimen, it is helpful to adopt this concept for understanding and investigating the principles of drug movements in the inner ear after local or systemic application. The LADME concept involves liberation, absorption, distribution, metabolism and elimination of drugs (fig. 1). While for whole body pharmacokinetics, the LADME processes are centered on blood circulation, in the ear they are centered on the inner ear fluids.

Liberation describes the release of the drug from its dosage form.

Absorption refers to the movement of the drug from the site of administration to the inner ear fluids (e.g. from the middle ear to the perilymph of the scala tympani (ST) through the round window membrane, RWM).

Distribution involves the processes by which the drug diffuses, flows or is transferred within and between the different fluid-filled compartments (perilymph and endolymph), and how it spreads from the fluid spaces into the various tissue compartments of the inner ear.

Metabolism is the chemical conversion or transformation of drugs into active moieties or compounds which are easier to eliminate.

Elimination describes the removal of the unchanged drug or metabolite from the inner ear (e.g. to blood, cerebrospinal fluid or the middle ear).

Although these processes generally follow the above sequence, they may occur simultaneously. While the drug is still being liberated from a controlled release for-

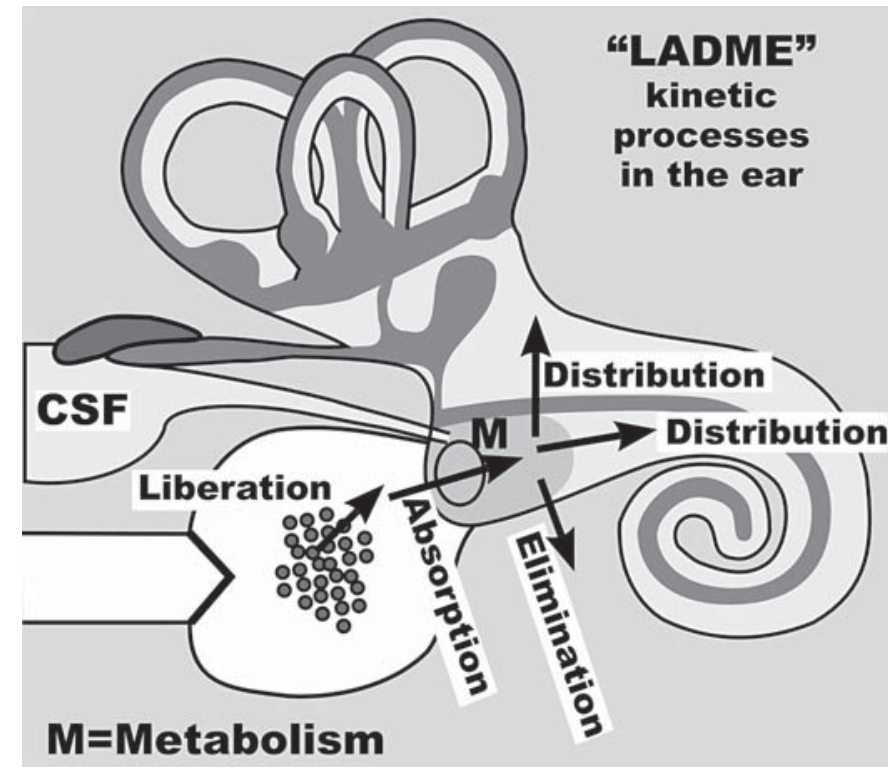

Fig. 1. Pharmacokinetic processes of the inner ear according to the LADME concept, as described for an intratympanic application of a formulated drug. Absorption occurs primarily through the round window membrane. The drug, upon entering the perilymph, distributes both within the scala tympani and into adjacent fluid and tissue-filled spaces. The drug is also subjected to metabolism and elimination to blood or CSF.

mulation, previously absorbed drug may already have been eliminated.

\section{Liberation}

Current efforts in the area of drug delivery in general, and also specifically in inner ear therapies, include the development of drugs which are liberated from a formulation over a period of time in a controlled manner. Types of sustained release formulations include liposomes, drug-loaded biodegradable microspheres and drug polymer conjugates, including gels [18-22]. Drug release from carrier systems may be driven only by the concentration gradient (such as for a drug in a resorbable gelatin sponge soaked with drug solution) or maintained by a gradual breakdown of the carrier, either spontaneously or induced by physical and chemical triggers (e.g. temperature or $\mathrm{pH}$ ), with subsequent release of drug.

\section{Absorption}

Absorption of a drug from the middle ear to perilymph of the inner ear can occur through a number of structures, including: 

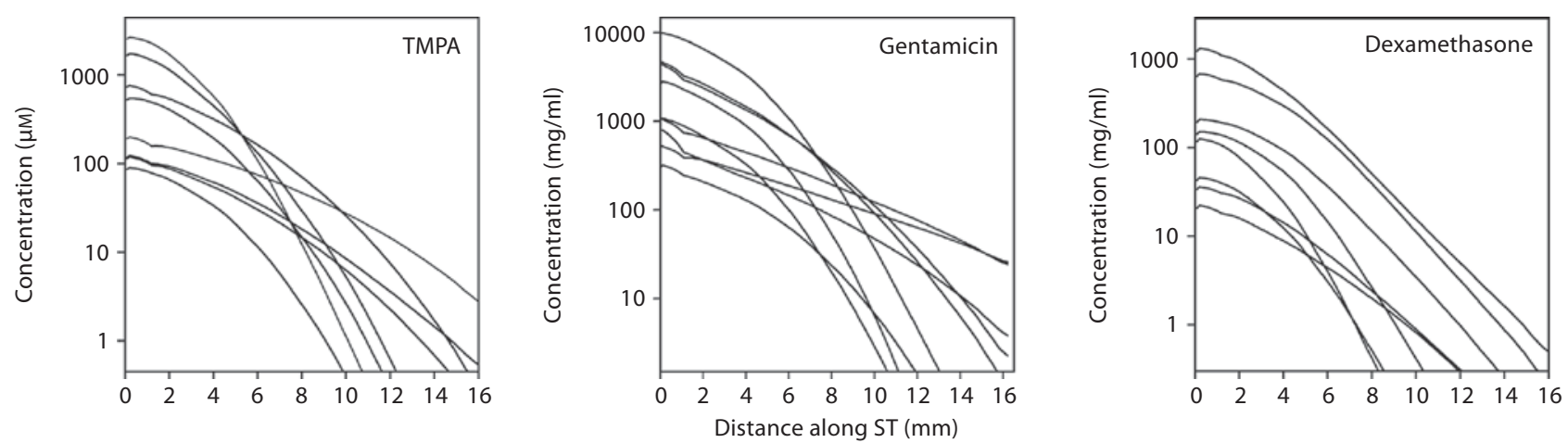

Fig. 2. Concentration gradients along ST of the guinea pig following 2- to 3-hour applications to the RWM. Distances are measured along the ST from the basal end. Results are shown for the marker ion TMPA [34], gentamicin [53] and dexamethasone [54]. In addition to the steep concentration gradients, it is also apparent that the basal turn $(0-2 \mathrm{~mm})$ concentrations of each substance vary by more than a factor of 10 , due to inter-animal variations in RWM permeability and elimination of the drug. Recently, it has been shown that these concentration gradients are stable with time [83].
Round Window Membrane. The RWM in humans, monkeys, felines and rodents consists of 3 main layers: (1) an outer epithelial layer facing the middle ear cavity; (2) a middle connective tissue layer; (3) an inner cellular layer facing the ST perilymph $[23,24]$. Tight junctions are present between cells of the outer layer, while in the middle layer fibroblasts, fibrocytes, collagen, elastin, capillaries, and myelinated and unmyelinated nerves have been described [25]. Many studies have demonstrated in qualitative terms that substances applied to the middle ear enter the basal turn of the ST, and may influence structure and function of the ear [24, 26-28]. In contrast, few have performed quantitative measurements of drug levels in the perilymph or measured RWM permeability. Of the pharmacokinetic studies in the literature, a substantial proportion cannot be interpreted quantitatively due to sampling methods that caused the fluid samples to be highly contaminated with CSF $[18,29,30,31]$. In these studies, large volumes $(10 \mu \mathrm{l})$ relative to the volume of the ST in the guinea pig $(4.6 \mu l)$ [32] were taken from the basal turn of ST. As the cochlear aqueduct enters ST at this location, samples taken nearby become severely contaminated with CSF that is drawn into the scala as the sample is aspirated. Based on measurements with marker ions, it was estimated that $10-\mu l$ samples taken from the basal turn of guinea pigs contained as little as $15 \%$ perilymph and $85 \%$ CSF [33]. Sample measurements are more readily interpreted when the samples are taken from a location further from the cochlear aqueduct. A better technique, in which multiple samples are taken sequentially from the cochlear apex within a period of a few minutes, allows both the concentration and the gradient of drug along the ST to be quantified [34]. Results obtained with this technique show that, following 2- to 3hour application of a drug or marker to the RWM, there are substantial gradients along the ST. The gradients determined for 3 substances are shown in figure 2 . For TMPA (trimethylphenylammonium: an ionic marker), gentamicin and dexamethasone, basal-apical concentration differences of over 1000-fold were found in most animals. The presence of basal-apical gradients following drug applications to the RWM is supported by a number of histological studies that suggested markers were at higher concentration or cellular damage was greater in the basal turn than in apical regions $[28,35,36]$. The concentration measurements in figure 2 also show that the basal turn concentration of drugs is variable, with over 10 -fold differences between animals being common. Measurements of entry rates using microdialysis have confirmed that the variability between animals arises from differences in RWM permeability [37]. RWM permeability has also been shown to be sensitive to experimental manipulations. Permeability is increased by local anesthetics [38], endotoxins and exotoxins [39, 40], histamine [41], drying through the use of suction near the round window niche [42], by osmotic disturbances and by the presence of benzyl alcohol (a commonly used preservative) in the applied solution [42]. 


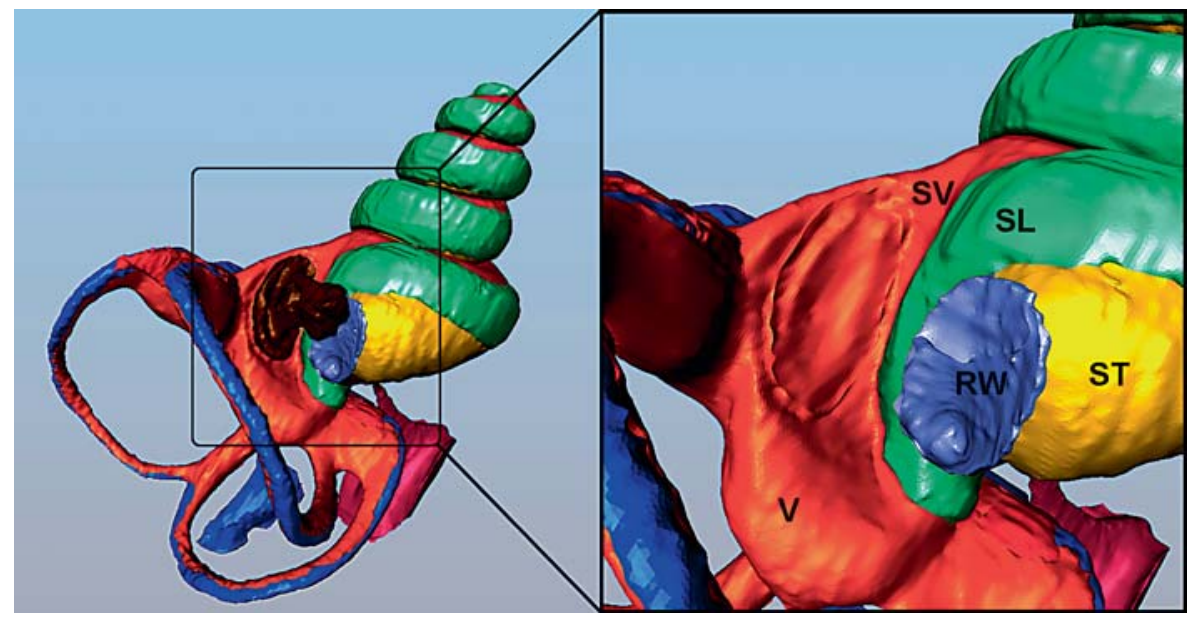

Fig. 3. Reconstructed 3D anatomy of the fluid spaces of the guinea pig inner ear derived by segmentation of an OPFOS (orthogonal-plane fluorescence optical sectioning) image set [84] using Amira software. The enlargement shows the basal turn with the stapes removed (leaving an imprint of the footplate). V = Vestibule; SL = spiral ligament; RW = round window. The SL follows the periphery of the RW almost half way around, providing a ma-

In addition, there is only limited knowledge about the individual processes of transmembrane transport contributing to substance absorption through the RWM, including passive diffusion, facilitated diffusion through carriers, active transport or phagocytosis.

Oval Window (Including the Stapes Footplate and Annular Ligament). Although a number of investigators have suggested substances may enter perilymph of the vestibule by this route $[36,43]$, it is technically difficult to measure the amount. Substances cross readily between the ST and scala vestibuli (SV) $[44,45]$, presumably passing through the spiral ligament (discussed further in 'Distribution'). Thus, the observation of drug or marker in the vestibule or saccule does not confirm that it entered through the oval window. In addition, attempts to occlude the RWM (such as with dental cement [36]) were found to be only partially effective. It is undoubtedly possible for drugs to pass through the thin bone of the stapes footplate or through the walls of the oval window niche in amounts which may be significant in the human. The amount of drug entering by this route, however, remains uncertain and likely depends on drug size and charge, but is thought to be small relative to that entering through the RWM.

The Bony Otic Capsule. It has recently been shown that when a drug is applied by filling the middle ear with solution in guinea pigs, the highest drug levels are produced jor route for drugs in the ST near the RW to diffuse across into the vestibule. This anatomic pathway accounts for how drugs applied intratympanically to the RW can gain access to vestibular structures. Blue = Endolymph; orange = perilymph of SV and V; yellow = perilymph of ST; green = spiral ligament; red = sensory structures; purple $=\mathrm{RW}$; magenta $=$ cochlear aqueduct; brown $=$ stapes. in the apical regions of the cochlea [46]. This results from the drug entering perilymph through the bony otic capsule, which is very thin in the apical turns of animals such as guinea pigs and chinchillas. This presents a considerable problem when studies in rodents are used as a model for drug delivery in the human, as it can be assumed that the thicker bone of the human otic capsule will represent a more effective boundary. The resulting drug distribution patterns along the length of the cochlea are therefore likely to differ markedly between animals and humans following intratympanic applications.

\section{Distribution}

The fluids of the inner ear show little evidence of 'stirring, i.e. no pronounced movement comparable to that of the systemic circulation. In the intact state, rates of volume flow of perilymph and endolymph are both exceedingly slow and the distribution of drugs within the fluid spaces is dominated by passive diffusion. Diffusion is a highly predictable process and its effects can be calculated with accuracy. The rate of substance movement by diffusion is nonlinear with distance, allowing drugs to spread rapidly over short distances (such as across a cochlear scala), but slowly over distances of more than a few millimeters. This results in large gradients along the cochlea when substances are applied to the basal turn, as shown earlier in figure 2. Also contributing to the gradi- 
ents along the length of the cochlea is the loss of drug from the scala as it diffuses. Losses result from the distribution into fluid spaces and tissue compartments adjacent to the scala, and through elimination from the ear to other much larger compartments, such as blood. For ST, substances readily distribute through the fluid spaces of the spiral ligament into the SV and the vestibule. Figure 3 shows the anatomy of the spiral ligament in the basal turn, which is seen to wrap around the periphery of approximately half the RWM. Drugs present in the perilymph at the base of ST close to the RWM would therefore be expected to have ready access to perilymph of the vestibule. In addition, drugs in the ST can pass through passages in the bone (canaliculi perforantes) into Rosenthal's canal and from there to the modiolus [47, 48], and into the fluid spaces of the organ of Corti [49]. Although the canaliculi perforantes in the medial wall of the ST appear rather large, data from experiments with neurotrophins suggest that only a small proportion of a drug infused into the ST reaches the vicinity of the spiral ganglion neuron cell bodies, suggesting that other barriers to diffusion may exist [50].

In addition, some substances may also enter endolymph, although this is likely to depend on the electrical charge of the molecule, which subjects it to the influence of cellular potentials and the endocochlear potential. Cationic markers are excluded from endolymph [44], while anionic markers are accumulated and retained there [51].

Apart from a drug's chemical characteristics, especially size and its hydrophobicity, distribution processes are primarily dependent on concentration gradients, so that the distribution of substances into different compartments of the inner ear can be reversed if the concentration gradient changes. This is apparent during sequential apical sampling, when CSF entering the basal turn of the ST gains drug as it passes through the scala towards the sampling site, and can be referred to as 'redistribution'. Indeed, the rate at which sequential sample concentrations decline during rapidly repeated sampling from the apex provides a valuable index of the rate and amount of drug available to redistribute into the scala during sampling.

It has also been suggested that protein levels in perilymph may be sufficient to bind or buffer some drugs [52]. Uptake of a drug into cellular structures of the ear may also occur. By causing a loss of drug from the scala, each of these processes acts to reduce the rate at which the drug spreads along the scala by diffusion. However, in contrast to the expected slowing of the rate of distribu- tion caused by these processes, the measured gradients along the ST in figure 2 could only be explained by the substances spreading towards the apex at a slightly faster rate than can be accounted for by diffusion. To account for the data, it was necessary to incorporate a low rate of apically directed perilymph flow into the simulations of the experiments. The perilymph flow rates averaged $0.019,0.021$ and $0.009 \mu \mathrm{l} / \mathrm{min}$ for TMPA [34], gentamicin [53] and dexamethasone [54], respectively. While these rates are extremely low, they suggest that perilymph flow may contribute significantly to drug distribution when its effects are accumulated over a period of hours or days.

\section{Metabolism}

Metabolism or biotransformation considers the transformation of the drug from one form to another with altered (lower or higher) effectiveness by tissues and enzymes within the ear or in adjacent compartments, such as the middle ear. There is, however, very limited quantitative data about the metabolism of specific groups of substances following their application to the inner ear. It has been shown that dexamethasone-phosphate is metabolized within the ear to the active moiety dexamethasone $[54,55]$. There is also accumulating evidence that extracellular purines in the cochlear fluids, important modulators of cochlear function and potential targets for manipulation [56], are regulated by ectonucleotidases [57].

\section{Elimination}

The rate of elimination of a drug from perilymph is one of the major factors influencing both the concentration achieved in perilymph and how far towards the apex a drug is distributed following its application to the RWM. For faster elimination rates, perilymph concentration saturates at a lower value and reaches a steady state more quickly than with slower elimination rates. In addition, when the amount of drug eliminated from a specific region becomes equal to the rate of diffusion along the scala at that point, then a steady state will be established and the drug will never reach a higher concentration at more apical locations. In order to determine how far a specific drug will spread along the cochlea, knowledge of the elimination rate is required. Determination of the elimination rate for a specific substance is, however, technically difficult. If drug is applied locally to the RWM, then the decline in concentration at a location near the application site (e.g. in the basal part of the ST) following the application results from the combined effects of both dis- 
Fig. 4. Idealized flowchart for drug delivery to the ear, taking into account the known distribution properties and limitations of different delivery systems. IT $=$ Intratympanic; SCC = semi-circular canal.

\section{Systematic Approach to Drug Delivery Strategies}

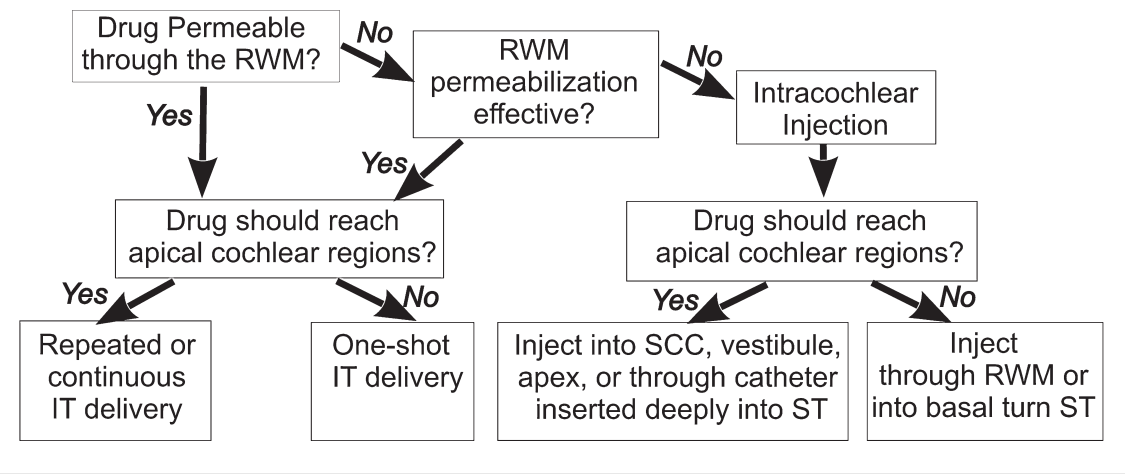

tribution and elimination, which would distort quantification of the elimination rate. If the time course of drug decline is monitored by taking samples repeatedly over a long period, such as samples taken hourly in the study by Parnes et al. [29], then the act of sampling, accompanied by drawing CSF into the cochlea, also contributes to the decline of drug concentration and prevents an accurate estimation of the elimination rate. Similarly, the use of microdialysis to follow perilymph concentration as a function of time $[37,58]$ does not provide a valid indication of elimination rate as the microdialysis procedure itself provides a major source of elimination [37]. As a result of these technical difficulties, there are relatively few studies from which drug elimination rates can be reliably derived. In a few studies, where all or most of the perilymph in the cochlea was taken as a single sample, the effects of redistribution were minimized. One series of studies investigated the kinetics of gentamicin in the chinchilla [59], in which large samples of perilymph (15 $\mu l)$ were taken from the vestibule. These studies were analyzed quantitatively [60] from which a perilymph elimination half-time of $500 \mathrm{~min}$ for gentamicin was determined. In another study, prednisolone kinetics were investigated in guinea pigs [61], obtaining perilymph by aspirating the entire contents of the ST and SV after the temporal bone was removed from the animal. Quantitative analysis of these data allowed an elimination half-life of $130 \mathrm{~min}$ for prednisolone to be derived [62]. These appear to be the only studies in which the experimental design allowed a meaningful interpretation of the elimination rate for drugs.

While pharmacokinetic analyses generally consider elimination of solutes from the scala fluids to blood, we know from anatomic data that this is an oversimplifica- tion. The major capillary beds in the ear are not located in, or directly associated with, the scalae. Although arterioles and venules run around the bony walls of SV and ST, respectively, they run within bony canals separated from the scala fluid spaces. Instead, the major capillary beds are associated with the spiral ganglion, the lateral wall and limited regions of the organ of Corti. Fluid pathways through the bone between the ST and the spiral ganglion, and between the SV and the modiolar spaces, have been demonstrated [47, 48]. Elimination of drugs from perilymph is therefore likely to occur indirectly, mediated by the vascularized tissues in communication with perilymph. Whether spiral ligament or modiolar sites dominate perilymph kinetics remains to be established. It is also likely that distribution of many substances will be influenced by active transport processes at physiological boundaries in the ear, comparable to those established for the eye [63], but given the complexities of pharmacokinetic studies of the ear, influences of specific transport processes on cochlear fluid pharmacokinetics have yet to be demonstrated.

\section{Delivery Systems and Protocols}

Based on knowledge of how drugs enter and are dispersed in the ear, it is possible to build a framework for how drugs can be delivered to the ear for specific purposes, as shown in figure 4. At present, the strategy for local drug delivery in clinical situations is dominated by intratympanic applications. For situations where the drug does not readily pass the RWM, or where better control of drug level is necessary, then intralabyrinthine applications are necessary. However, none of the intralaby- 
rinthine application techniques used in animals has yet been proven safe enough for use in humans, although this field is advancing rapidly.

\section{Intratympanic (Middle Ear) Applications}

Delivery protocols used in conjunction with intratympanic applications have included single injections (a 'oneshot'), repeated injections ( 3 times a day over a period of time, or longer intervals such as weekly), or continuous delivery over a period of days using a microcatheter. The influence of delivery protocol on the amount and time course of drug in perilymph has not yet been documented, but calculations show that a key variable influencing the perilymph concentration achieved by each application is the time the drug remains in contact with the RWM [37]. As most of the decline in drug level near the application site results from distribution of the drug into other parts of the ear, longer application times help maintain the drug level while nearby regions become loaded with the drug. In clinical terms, this means that to reduce variability of perilymph drug levels to a minimum, the time the drug remains in the round window niche must be controlled as closely as possible. Calculations also show that multiple injection or continuous application protocols not only produce higher drug concentrations near the application site, but also allow proportionately greater concentrations to reach higher turns of the cochlea [64]. The application protocol can therefore be modified according to the goals of the local drug application. For treatment of Ménière's patients with gentamicin, where the goal is to suppress the balance system while minimizing gentamicin-induced hearing loss, a one-shot application is most appropriate, as this results in the steepest drug gradient along the cochlea, minimizing the gentamicin concentration reaching cochlear locations responsible for speech frequencies. In contrast, when the goal is to distribute the drug throughout the cochlea, then multiple applications per day or continuous delivery protocols would minimize longitudinal gradients to the greatest degree possible. There are numerous other factors that influence the perilymph drug level resulting from a specific application protocol, including the RWM permeability to the drug and how fast the drug is eliminated from the round window niche by the middle ear mucosa, through drainage to the spaces of the temporal bone, and by the injected solution leaving the middle ear through the eustachian tube.

\section{Intralabyrinthine Applications}

In the literature, the ambiguous terms 'infusion', 'perfusion' and 'injection' have been used to describe the de- livery of drugs to the inner ear. The term 'perfusion' of the ear was originally used to describe the passage of solution through the perilymphatic spaces from a site of entry to an open outlet. Perfusion was, however, also used to describe intratympanic application without perforation of the otic capsule, i.e. the solution passing solution across the ear $[14,65]$. 'Infusion' (derived from the Latin verb 'to pour into') also suggests a passive slow delivery of solution to the ear. In contrast, the terms 'intralabyrinthine injection' and 'intracochlear injection' are preferred as they more accurately represent a more rapidly occurring and forceful introduction of solution directly into the fluid spaces of the inner ear.

In general pharmacokinetics, the term bioavailability is commonly used to describe the rate and extent of drug input. For extracochlear applications, such as intratympanic injections to the round window niche, the percentage of drug entering the inner ear (bioavailability) is relatively low. It was estimated that the basal turn concentration only reached a mean of $2.5 \%$ of the applied gentamicin concentration and $1.4 \%$ of the applied dexamethasone-phosphate concentration with a round window irrigation protocol and $0.17 \%$ of the applied gentamicin concentration when the bulla was filled with solution $[46,53]$. Drugs injected directly into the perilymph would theoretically be expected to exhibit $100 \%$ bioavailability, and in this respect are comparable to an intravenous application when considering the entire human body. In reality, $100 \%$ bioavailability might not be reached in all intralabyrinthine application techniques due to leaks either at the application site or at sites of fluid efflux, such as the cochlear aqueduct. Nevertheless, bioavailability of drug within the inner ear can be substantially increased by direct application of the drug through the RWM, through the stapes footplate, into the lateral semicircular canals or into the endolymphatic sac.

Under conditions when the injection pipette is sealed into the otic capsule, intralabyrinthine injections produced more consistent perilymph concentrations (of the marker ion) than applications to the round window niche [66]. Difficulties arise, however, when there is any form of leak at the injection site or when an outlet for the injected solution is provided. For the same reasons that perforations of the otic capsule cause contamination of fluid samples with CSF, perforations to insert an injection pipette cause a release of intracochlear pressure that results in an artifactual volume flow from the cochlear aqueduct entering the ST in the basal turn to the site of the perforation. In guinea pigs, CSF entry rates of $0.5-1 \mu \mathrm{l} / \mathrm{min}$ occur, which is fast enough to displace drug solution from 
Fig. 5. Schematic of the balance between the control of perilymph drug level by different application approaches and the risk to the patient's hearing and/or balance by the drug application alone. Intratympanic drug applications are low risk, but produce variable perilymph levels. Improved control of drug levels may be achieved by manipulations of RWM permeability (arrows). Intracochlear drug injections give better control of perilymph drug concentration, but the safety of such procedures has not been demonstrated. Delivering drugs from a cochlear implant may carry little additional risk to the patient. As experience is gained, the goal remains to provide quantitative control of the drug level in perilymph while minimizing risk to hearing and balance function.

\section{Clinical Trade-off Between Control of Drug Level and Risk}

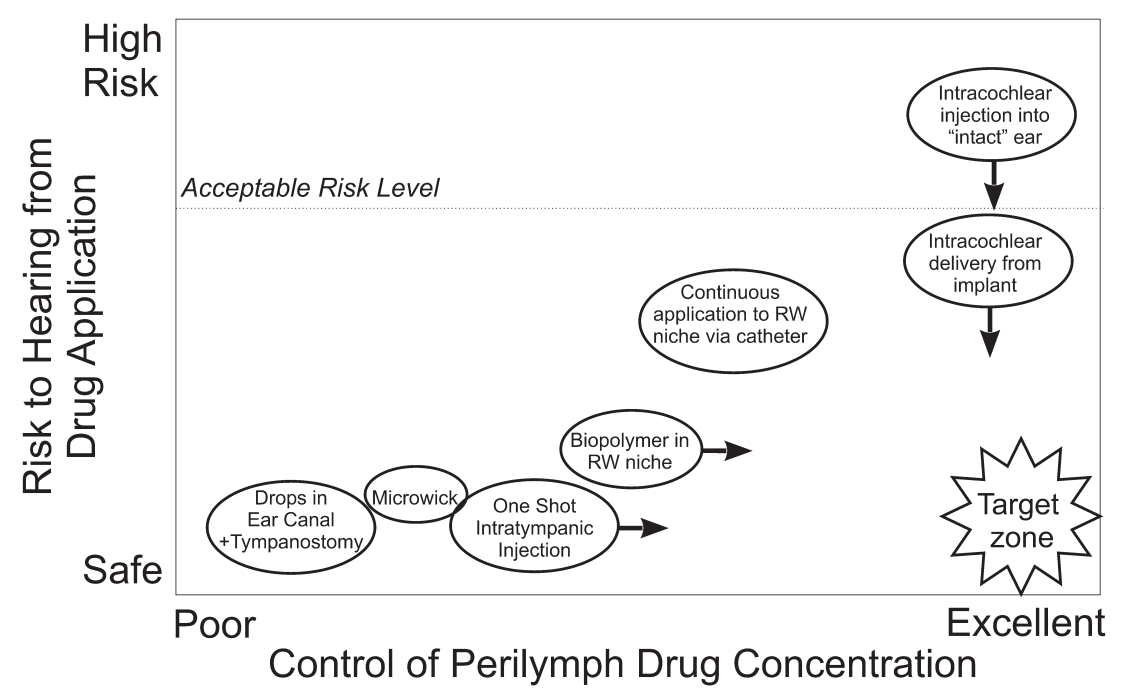

the ear (especially from the basal turn of ST, where CSF enters) within minutes. Attempts to seal injection sites through the bone with bone dust, adipose tissue or fascia may be effective after a number of hours or days as the site scars, but are unlikely to provide an immediate fluidtight seal. Thus, the procedures used to seal the injection site may critically influence the perilymph drug level. In humans, the situation may be considerably different from animal models as the cochlear aqueduct is narrower and CSF pressure at the level of the aqueduct may be negative when the patient is sitting or standing.

The spread of drug from the site of injection depends on the rate of injection and the site (if any) where the displaced perilymph exits the ear. For low injection rates, the induced rate of perilymph flow may be low, and spread from the injection site will be dominated by diffusion. This has been demonstrated both by realtime measurements of markers [67] and by functional measures during pulsed injections into the basal turn of the ST [68]. For higher injection rates, the spread of drug can only be predicted if the fluid outlet site is known. As a result of these factors, the region(s) of the inner ear affected by intralabyrinthine injections depends to a large extent on the site of injection. Possible injection sites (as shown in fig. 4) include the RWM (through fenestrations in the bone in the basal turn of ST), the stapes footplate or the semi-circular canals, and the endolymphatic sac.
Many studies have reported the injection of agents through the RWM using hand-held narrow-gauge hypodermic syringes. However, when perilymph concentration was measured following injections through the RWM with fine (20- $\mu$ M OD) bevelled pipettes held in a micromanipulator, small leaks around the pipettes caused significant washout of the drug [67]. It was calculated that the leakage rate under these conditions averaged just 90 $\mathrm{nl} / \mathrm{min}$, which was sufficient to wash out the drug but was not visible with an operating microscope. The leakage around the pipette was markedly reduced when the round window niche was filled with Healon gel before the injection pipette was inserted. These results suggest that injections through the RWM with handheld hypodermic syringes are likely to result in even higher rates of perilymph leakage, and cannot be regarded as a quantitative delivery method.

\section{Cochlear Implants}

There is accumulating evidence from animal experiments that locally applied glucocorticoids can help preserve acoustic hearing thresholds following cochlear implantation [69-71]. As there is increasing interest in the possibility of implanting patients with high-frequency hearing loss but good low-frequency hearing, it has become an important goal to perform the implantation with minimal damage to residual hearing [72-74]. Other drug candidates include the use of neurotrophins 
to preserve spiral ganglion cells [75] and apoptosis inhibitors to minimize insertion trauma [76]. There are several possible strategies of intracochlear drug delivery in combination with cochlear implants, including applications to the RWM prior to surgery [77], one-shot injections into the ST at the time of implantation [78, 79], 'bathing' the electrode in drug solution or a gel preparation prior to insertion into the cochlea, drug release from the electrode carrier itself which also functions as a scaffold, drug release from a reservoir in the electrode carrier, drug injection through an incorporated channel attached to a pump [80,81], or by surface coating of the electrode carrier with a controlled-release formulation [82].

\section{Clinical Considerations}

At present, the most widely used approach to deliver drugs locally to the inner ear is through the use of intratympanic applications. This is in large part because of the requirement for a safe and feasible way of drug delivery to the inner ear, especially to minimize damage to hearing, which became a major component of local gentamicin applications in the treatment of Ménière's disease. Animal studies, however, showed that perilymph drug levels achieved with RWM applications are highly variable $[29,37,54,61]$ and that more consistent perilymph concentrations can be achieved by intracochlear injec- tions of substances [67]. However, based on experience with surgical procedures that involve perforation of the inner ear such as stapedectomy or the cochlear implantation of patients with residual hearing, it is well known that perforation and/or surgical manipulation of the ear carries a significant risk of deafness. Appropriate techniques for the intralabyrinthine injections of drugs have not yet been developed and proven safe. This results in a balance or trade-off between the ability of the delivery method to control the perilymph drug level and the risk of the drug delivery procedure to the hearing and balance function of the patient as schematized in figure 5. In order to have good control of perilymph drug levels and effective drug delivery without damage to the ear, we either need to reduce the variability and increase the bioavailability associated with intratympanic applications (such as by better control of the drug level in the round window niche or by permeabilizing the RWM) or we need to develop safe methods of intracochlear drug delivery that do not damage the ear.

\section{Acknowledgements}

This study was supported by research grant RO1 DC01368 (to A.N.S.) from the National Institute on Deafness and other Communication Disorders (NIDCD), National Institutes of Health (NIH), and by grants 0313844 B and 0314103 (to S.K.P.) from the Federal Ministry of Education and Research (BMBF), Germany.

\section{References}

1 Ersner MS, Spiegel EA, Alexander MH: Transtympanic injection of anesthetics for the treatment of Ménière's syndrome. AMA Arch Otolaryngol 1951;54:43-52.

2 Völger G: Beseitigung von Labyrinthliquordruckstörungen bei dem Menièrschen Symptomkomplex durch das Hyaluronidasepräparat Kinetin (in German). HNO 1952; 3:142-147.

13 Schuknecht HF: Ablation therapy for the relief of Ménière's disease. Laryngoscope 1956; 66:859-870.

4 Lange G: Gentamicin and other ototoxic antibiotics for the transtympanic treatment of Menière's disease. Arch Otorhinolaryngol 1989;246:269-270.

5 Blakley BW: Clinical forum: a review of intratympanic therapy. Am J Otol 1997;18: 520-526.
6 Carey J: Intratympanic gentamicin for the treatment of Meniere's disease and other forms of peripheral vertigo. Otolaryngol Clin North Am 2004;37:1075-1090.

-7 Schwab B, Lenarz T, Heermann R: Use of the round window micro cath for inner ear therapy - results of a placebo-controlled, prospective study on chronic tinnitus (in German). Laryngorhinootologie 2004;83:164172.

-8 Van Wijk F, Staecker H, Keithley E, Lefebvre PP: Local perfusion of the tumor necrosis factor alpha blocker infliximab to the inner ear improves autoimmune neurosensory hearing loss. Audiol Neurootol 2006;11:357365.

-9 Suckfuell M, Canis M, Strieth S, Scherer H, Haisch A: Intratympanic treatment of acute acoustic trauma with a cell-permeable JNK ligand: a prospective randomized phase I/II study. Acta Otolaryngol 2007;127:938-942.
10 Barrs DM: Intratympanic corticosteroids for Meniere's disease and vertigo. Otolaryngol Clin North Am 2004;37:955-972.

-11 Rauch SD: Intratympanic steroids for sensorineural hearing loss. Otolaryngol Clin North Am 2004;37:1061-1074.

$\checkmark 12$ Haynes DS, O’Malley M, Cohen S, Watford $\mathrm{K}$, Labadie RF: Intratympanic dexamethasone for sudden sensorineural hearing loss after failure of systemic therapy. Laryngoscope 2007;117:3-15.

13 Plontke SK, Löwenheim H, Mertens J, Engel C, Meisner C, Weidner A, Zimmermann R, Preyer S, Koitschev A, Zenner HP: Randomized, double blind, placebo controlled trial on the safety and efficacy of continuous intratympanic dexamethasone delivered via a round window catheter for severe to profound sudden idiopathic sensorineural hearing loss after failure of systemic therapy. Laryngoscope 2009;119:359-369. 
14 Light JP, Silverstein H: Transtympanic perfusion: indications and limitations. Curr Opin Otolaryngol Head Neck Surg 2004;12: 378-383.

$\checkmark 15$ Sakata E, Ito Y, Itoh A: Clinical experiences of steroid targeting therapy to inner ear for control of tinnitus. Int Tinnitus J 1997;3:117121.

16 Harris JP: Inner ear steroid perfusion. Curr Opin Otolaryngol Head Neck Surg 2007;15: 303.

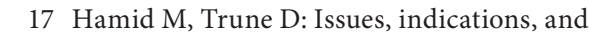
controversies regarding intratympanic steroid perfusion. Curr Opin Otolaryngol Head Neck Surg 2008;16:434-440.

-18 Arnold W, Senn P, Hennig M, Michaelis C, Deingruber K, Scheler R, Steinhoff HJ, Riphagen F, Lamm K: Novel slow- and fasttype drug release round-window microimplants for local drug application to the cochlea: an experimental study in guinea pigs. Audiol Neurootol 2005; 10:53-63.

-19 Endo T, Nakagawa T, Kita T, Iguchi F, Kim TS, Tamura T, Iwai K, Tabata Y, Ito J: Novel strategy for treatment of inner ears using a biodegradable gel. Laryngoscope 2005;115: 2016-2020.

20 Tamura T, Kita T, Nakagawa T, Endo T, Kim TS, Ishihara T, Mizushima Y, Higaki M, Ito J: Drug delivery to the cochlea using PLGA nanoparticles. Laryngoscope 2005; 115 : 2000-2005.

-21 Ge X, Jackson RL, Liu J, Harper EA, Hoffer ME, Wassel RA, Dormer KJ, Kopke RD, Balough BJ: Distribution of PLGA nanoparticles in chinchilla cochleae. Otolaryngol Head Neck Surg 2007;137:619-623.

22 Paulson DP, Abuzeid W, Jiang H, Oe T, O'Malley BW, Li D: A novel controlled local drug delivery system for inner ear disease. Laryngoscope 2008;118:706-711.

23 Goycoolea MV, Lundman L: Round window membrane. Structure function and permeability: a review. Microsc Res Tech 1997;36: 201-211.

24 Goycoolea MV: Clinical aspects of round window membrane permeability under normal and pathological conditions. Acta Otolaryngol 2001;121:437-447.

-25 Schachern PA, Paparella MM, Duvall AJ 3rd: The normal chinchilla round window membrane. Arch Otolaryngol 1982;108:550-554.

-26 Rahm WE, Strother WF, Gulick WL, Crump JF: The effects of topical anesthetics upon the ear. Ann Otol Rhinol Laryngol 1959;68: 1037-1046.

27 Imamura S, Adams JC: Distribution of gentamicin in the guinea pig inner ear after local or systemic application. J Assoc Res Otolaryngol 2003;2:176-195.

-28 Wagner N, Cayé-Thomasen P, Laurell G, Bagger-Sjöbäck D, Thomsen J: Cochlear hair cell loss in single-dose versus continuous round window administration of gentamicin. Acta Otolaryngol 2005;125:340-345.
29 Parnes LS, Sun AH, Freeman DJ: Corticosteroid pharmacokinetics in the inner ear fluids: an animal study followed by clinical application. Laryngoscope 1999;109:1-17.

30 Liu HJ, Dong MM, Chi FL: Dexamethasone pharmacokinetics in guinea pig inner ear perilymph. ORL J Otorhinolaryngol Relat Spec 2006;68:93-98.

31 Yang J, Wu H, Zhang P, Hou DM, Chen J, Zhang SG: The pharmacokinetic profiles of dexamethasone and methylprednisolone concentration in perilymph and plasma following systemic and local administration. Acta Otolaryngol 2008;128:496-504.

- 32 Thorne M, Salt AN, DeMott JE, Henson MM, Henson OW, Gewalt SL: Cochlear fluid space dimensions for six species derived from reconstructions of three-dimensional magnetic resonance images. Laryngoscope 1999;109:1661-1668.

33 Salt AN, Kellner C, Hale S: Contamination of perilymph sampled from the basal cochlear turn with cerebrospinal fluid. Hear Res 2003; 182:24-33.

34 Mynatt R, Hale SA, Gill RM, Plontke SKR, Salt AN: Demonstration of a longitudinal concentration gradient along scala tympani by sequential sampling of perilymph from the cochlear apex. J Assoc Res Otolaryngol 2006; 7:182-193.

35 Stöver T, Yagi M, Raphael Y: Cochlear gene transfer: round window versus cochleostomy inoculation. Hear Res 1999;136:124130.

36 Saijo S, Kimura RS: Distribution of HRP in the inner ear after injection into the middle ear cavity. Acta Otolaryngol 1984;97:593610.

37 Hahn H, Kammerer B, DiMauro A, Salt AN, Plontke S: Cochlear microdialysis for quantification of dexamethasone and fluorescein entry into scala tympani during round window administration. Hear Res 2006;212: 236-244.

38 Höft J: The permeability of the round window membrane and its changes by pantocaine (tetracaine). Arch Klin Exp Ohren Nasen Kehlkopfheilkd 1969;193:128-137.

-39 Ikeda K, Morizono T: Changes of the permeability of round window membrane in otitis media. Arch Otolaryngol Head Neck Surg 1988;114:895-897.

40 Selivanova O, Maurer J, Ecke U, Mann WJ: The effects of Streptolysin-O and sodium hyaluronate on the permeability of the round window membrane in guinea pigs - an electrophysiologic study (in German). Laryngorhinootologie 2003;82:235-239.

41 Chandrasekhar SS, Rubinstein RY, Kwartler JA, Gatz M, Connelly PE, Huang E, Baredes S: Dexamethasone pharmacokinetics in the inner ear: comparison of route of administration and use of facilitating agents. Otolaryngol Head Neck Surg 2000;122:521-528.
42 Mikulec AA, Hartsock JJ, Salt AN: Permeability of the round window membrane is influenced by the composition of applied drug solutions and by common surgical procedures. Otol Neurotol 2008;29:1020-1026.

43 Tanaka K, Motomura S: Permeability of the labyrinthine windows in guinea pigs. Arch Otorhinolaryngol 1981;233:67-73.

44 Salt AN, Ohyama K, Thalmann R: Radial communication between the perilymphatic scalae of the cochlea. I. Estimation by tracer perfusion. Hear Res 1991;56:29-36.

45 Salt AN, Ohyama K, Thalmann R: Radial communication between the perilymphatic scalae of the cochlea. II. Estimation by bolus injection of tracer into the sealed cochlea. Hear Res 1991;56:37-43.

-46 Mikulec AA, Plontke SK, Hartsock JJ, Salt AN: Entry of substances into perilymph through the bone of the otic capsule after intratympanic applications in guinea pigs: implications for local drug delivery in humans. Otol Neurotol 2009;30:131-138.

-47 Shepherd RK, Colreavy MP: Surface microstructure of the perilymphatic space: implications for cochlear implants and cell- or drug-based therapies. Arch Otolaryngol Head Neck Surg 2004;130:518-523.

48 Rask-Andersen H, Schrott-Fischer A, Pfaller K, Glueckert R: Perilymph/modiolar communication routes in the human cochlea. Ear Hear 2006;27:457-465.

49 Ilberg C, Vosteen KH: Permeability of the in ner ear membranes. Acta Otolaryngol 1969; 67:165-170.

50 Richardson RT, O'Leary S, Wise A, Hardman J, Clark G: A single dose of neurotrophin-3 to the cochlea surrounds spiral ganglion neurons and provides trophic support. Hear Res 2005;204:37-47.

51 Salt AN, DeMott JE: Endolymph volume changes during osmotic dehydration measured by two marker techniques. Hear Res 1995;90:12-23.

52 Swan EE, Mescher MJ, Sewell WF, Tao SL, Borenstein JT: Inner ear drug delivery for auditory applications. Adv Drug Deliv Rev 2008;60:1583-1599.

53 Plontke SK, Mynatt R, Gill RM, Salt AN: Concentration gradient along scala tympani following the local application of gentamicin to the round window membrane. Laryngoscope 2007;117:1191-1198.

54 Plontke SK, Biegner T, Kammerer B, Delabar U, Salt AN: Dexamethasone concentration gradients along scala tympani after application to the round window membrane. Otology Neurotol 2008;29:401-406.

55 Hargunani CA, Kempton JB, DeGagne JM, Trune DR: Intratympanic injection of dexamethasone: time course of inner ear distribution and conversion to its active form. Otol Neurotol 2006;27:564-569.

56 Housley GD: Extracellular nucleotide signaling in the inner ear. Mol Neurobiol 1998; $16: 21-48$ 
57 Vlajkovic SM, Housley GD, Muñoz DJ, Robson SC, Sévigny J, Wang CJ, Thorne PR: Noise exposure induces up-regulation of ecto-nucleoside triphosphate diphosphohydrolases 1 and 2 in rat cochlea. Neuroscience 2004; 126:763-773.

58 Hibi T, Suzuki T, Nakashima T: Perilymphatic concentration of gentamicin administered intratympanically in guinea pigs. Acta Otolaryngol 2001;121:336-341.

- 59 Hoffer ME, Balough B, Kopke RD, et al: Morphologic changes in the inner ear of Chinchilla laniger after middle ear administration of gentamicin in a sustained-release vehicle. Otolaryngol Head Neck Surg 1999; 120:643-648.

60 Plontke SK, Wood AW, Salt AN: Analysis of gentamicin kinetics in fluids of the inner ear with round window administration. Otol Neurotol 2002;23:967-974.

-61 Bachmann G, Su J, Zumegen C, Wittekindt C, Michel O: Permeabilität der runden Fenstermembran für Prednisolon-21-Hydrogensuccinat. HNO 2001;49:538-542.

62 Plontke SK, Salt AN: Quantitative interpretation of corticosteroid pharmacokinetics in inner ear fluids using computer simulations. Hear Res 2003;182:34-42.

63 Hughes PM, Olejnik O, Chang-Lin JE, Wilson CG: Topical and systemic drug delivery to the posterior segments. Adv Drug Deliv Rev 2005;57:2010-2032.

-64 Salt AN, Gill RM, Plontke SK: Dependence of hearing changes on the dose of intratympanically-applied gentamicin: a metaanalysis using mathematical simulations of clinical drug delivery protocols. Laryngoscope 2008;118:1793-1800.

-65 Shea JJ Jr: The role of dexamethasone or streptomycin perfusion in the treatment of Meniere's disease. Otolaryngol Clin North Am 1997;30:1051-1059.

66 Salt AN, Hale SA, Plonkte SK: Perilymph sampling from the cochlear apex: a reliable method to obtain higher purity perilymph samples from scala tympani. J Neurosci Methods 2006;153:121-129.
67 Salt AN, Sirjani DB, Hartsock JJ, Gill RM, Plontke SK: Marker retention in the cochlea following injections through the round window membrane. Hear Res 2007;232:78-86.

68 Chen Z, Kujawa SG, McKenna MJ, Fiering JO, Mescher MJ, Borenstein JT, Swan EE, Sewell WF: Inner ear drug delivery via a reciprocating perfusion system in the guinea pig. J Control Release 2005;110:1-19.

69 Ye Q, Tillein J, Hartmann R, Gstoettner W, Kiefer J: Application of a corticosteroid (Triamcinolon) protects inner ear function after surgical intervention. Ear Hear 2007;28:361369.

70 James DP, Eastwood H, Richardson RT, O'Leary SJ: Effects of round window dexamethasone on residual hearing in a guinea pig model of cochlear implantation. Audiol Neurootol 2008;13:86-96.

-71 Vivero RJ, Joseph DE, Angeli S, He J, Chen S, Eshraghi AA, Balkany TJ, Van de Water TR: Dexamethasone base conserves hearing from electrode trauma-induced hearing loss. Laryngoscope 2008;118:2028-2035.

72 Lehnhardt E: Intracochlear placement of cochlear implant electrodes in soft surgery technique HNO (in German). 1993;41:356359.

73 Kiefer J, Gstoettner W, Baumgartner W, Pok SM, Tillein J, Ye Q, von Ilberg C: Conservation of low-frequency hearing in cochlear implantation. Acta Otolaryngol 2004;124: 272-280.

74 Gantz BJ, Turner C, Gfeller KE, Lowder MW: Preservation of hearing in cochlear implant surgery: advantages of combined electrical and acoustical speech processing. Laryngoscope 2005; 115:796-802.

75 Richardson RT, Thompson B, Moulton S, Newbold C, Lum MG, Cameron A, Wallace G, Kapsa R, Clark G, O'Leary S: The effect of polypyrrole with incorporated neurotrophin-3 on the promotion of neurite outgrowth from auditory neurons. Biomaterials 2007;28:513-523.

76 Eshraghi AA, Wang J, Adil E, He J, Zine A, Bublik M, Bonny C, Puel JL, Balkany TJ, Van De Water TR: Blocking c-Jun-N-terminal kinase signaling can prevent hearing loss induced by both electrode insertion trauma and neomycin ototoxicity. Hear Res 2007; 226:168-177.
77 Bird PA, Begg EJ, Zhang M, Keast AT, Murray $\mathrm{DP}$, Balkany $\mathrm{TJ}$ : Intratympanic versus intravenous delivery of methylprednisolone to cochlear perilymph. Otol Neurotol 2007;28: 1124-1130.

78 Kiefer J, Gstoettner W, Baumgartner W, Pok SM, Tillein J, Ye Q, von Ilberg C: Conservation of low-frequency hearing in cochlear implantation. Acta Otolaryngol 2004;124: 272-280.

79 Paasche G, Tasche C, Stöver T, LesinskiSchiedat A, Lenarz T: The long-term effects of modified electrode surfaces and intracochlear corticosteroids on postoperative impedances in cochlear implant patients. Otol Neurotol 2009;30:592-598.

80 Garnham C, Reetz G, Jolly C, Miller J, Salt A, Beal F: Drug delivery to the cochlea after implantation: consideration of the risk factors. Cochlear Implants Int 2005; 6(suppl 1):1214.

81 Hochmair I, Nopp P, Jolly C, Schmidt M, Schösser H, Garnham C, Anderson I: MEDEL cochlear implants: state of the art and a glimpse into the future. Trends Amplif 2006; 10:201-219.

82 Richardson RT, Wise AK, Thompson BC, Flynn BO, Atkinson PJ, Fretwell NJ, Fallon JB, Wallace GG, Shepherd RK, Clark GM, O'Leary SJ: Polypyrrole-coated electrodes for the delivery of charge and neurotrophins to cochlear neurons. Biomaterials 2009;30: 2614-2624.

83 Hahn H, Biegner T, Kammerer B, Delabar U, Salt AN, Plontke SK: Persistence of dexamethasone drug levels and base to apex gradients in the perilymph of the scala tympani after single shot injections through the round window membrane (abstract). J Assoc Res Otolaryngol 2009;32:115.

84 Voie AH: Imaging the intact guinea pig tym panic bulla by orthogonal-plane fluorescence optical sectioning microscopy. Hear Res 2002;171:119-128. 